\title{
An X-Ray Diffraction Study of Atomic Ordering in Platinum-Rich Copper-Platinum Alloys
}

\author{
By Nan-Chung Wu*, Hiroshi Iwasaki** and Shiro Ogawa**
}

\begin{abstract}
Atomic ordering in the copper-platinum alloy system has been studied by $\mathrm{X}$-ray diffraction using single crystals with compositions of $\mathrm{Cu}-48,54,61,66$ and 70 at $\% \mathrm{Pt}$. At the composition near the equiatomic one, an ordered structure $\mathrm{CuPt}$, first found by Johansson and Linde, has been confirmed. A negative result has been obtained for the existence of an ordered structure $\mathrm{Cu}_{3} \mathrm{Pt}_{5}$, which was proposed by Linde. An ordered structure formed at the composition near $\mathrm{CuPt}_{3}$ has been shown to be consistent with the structure model of Tang rather than that of Schneider and Esch.
\end{abstract}

(Received November 18, 1972)

\section{Introduction}

Copper and platinum are known to form a continuous alloy solid solution in the whole composition range. Johansson and Linde ${ }^{(1)}$ found the existence of ordered atomic arrangements in fully annealed solid solutions by means of $\mathrm{X}$-ray diffraction. At the equiatomic composition, an ordered arrangement, designated as CuPt, is such that (111) layers of the face-centered cubic lattice are occupied alternately by copper and platinum atoms, as shown in Fig. 1.

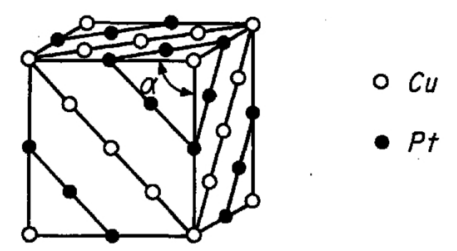

(a)
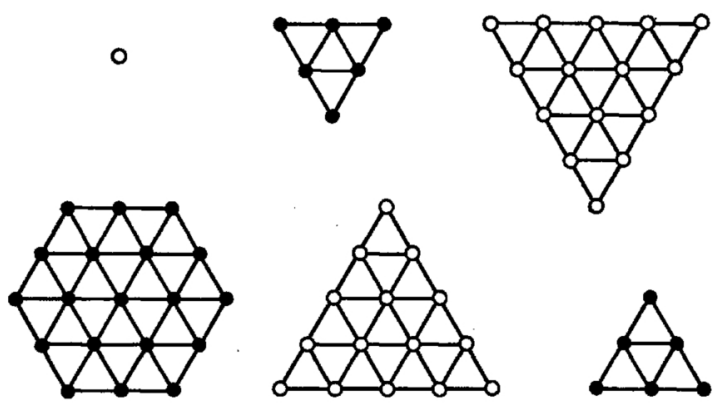

(b)

Fig. 1 Atomic arrangement of the ordered structure of CuPt. At the top of the figure is shown a conventional unit cell containing 32 atoms. Angle $\alpha$ is slightly larger than $90^{\circ}$. The atomic arrangement on successive (111) layers is shown below.

* Graduate School, Tohoku University, Sendai, Japan. Present address: Union Industrial Research Institute, 1021 Kuang Fu Road, Hsinchu, Taiwan, Republic of China.

** The Research Institute for Iron, Steel and Other Metals, Tohoku University, Sendai, Japan.

(1) C. H. Johansson and I. O. Linde: Ann. Phys., 82 (1927), 459.

Trans. JIM
A resulting symmetry of the crystal is rhombohedral and the original cubic lattice is slightly distorted in the [111] direction. The ordered phase with this type of atomic arrangement was found to exist in a wide composition ragne centered at $50 \% \mathrm{Pt}^{\dagger}$. Linde $^{(2)}$ reported that at the composition of about $65 \% \mathrm{Pt}$, excess platinum atoms replace copper atoms not at random but in a regular fashion. This ordered arrangement, shown in Fig. 2, has a rhombohedral symmetry and is designated as $\mathrm{Cu}_{3} \mathrm{Pt}_{5}$ or CuPt II. Schneider and $\operatorname{Esch}^{(3)}$ made an extensive study of the present alloy system and obtained the following results; (a) the ordered atomic arrangement proposed by Linde was not observed by their experiment. This was supported by a measurement of the composition dependence of electrical conductivity. (b) At $72.5 \% \mathrm{Pt}$, an ordered phase designated as $\mathrm{CuPt}_{3}$ was found to
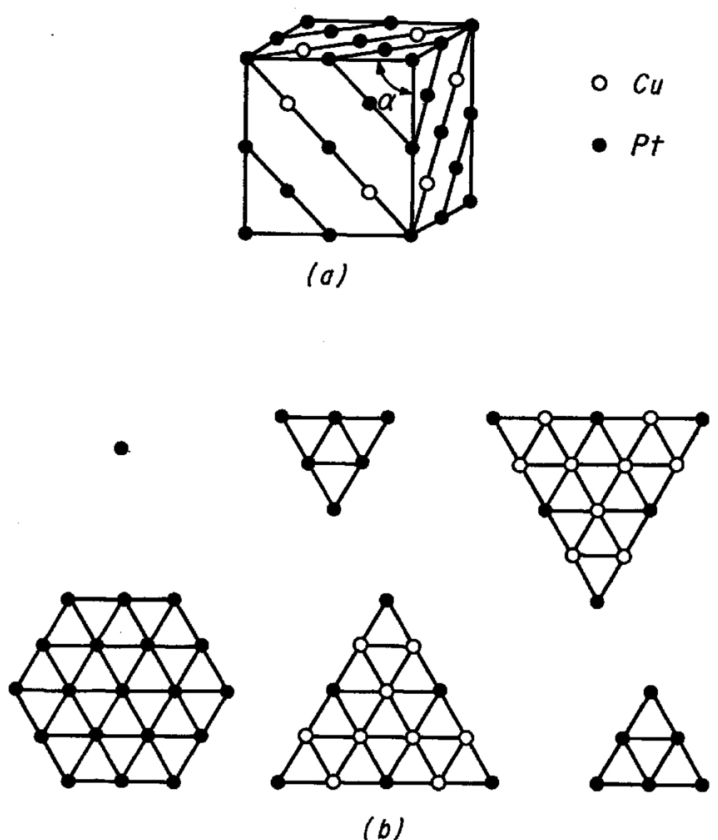

Fig. 2 Atomic arrangement of the structure model of $\mathrm{Cu}_{3} \mathrm{Pt}_{5}$ proposed by Linde.

$\dagger$ All percentages are referred to atomic percent in the present paper.

(2) I. O. Linde: Ann. Phys., [5] 30 (1937), 151.

(3) A. Schneider and U. Esch: Z. Elektrochem., 50 (1944), 290.

1973 Vol. 14 


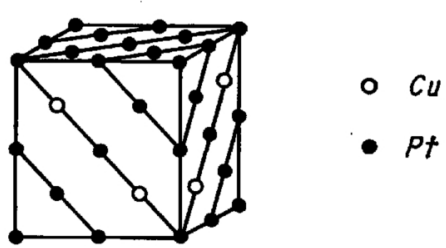

(a)
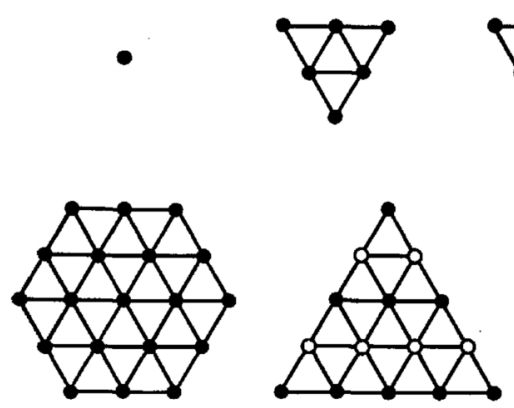

(b)

Fig. 3 Atomic arrangement of the structure model of $\mathrm{CuPt}_{3}$ proposed by Schneider and Esch.

exist. This phase has an atomic arrangement having two kinds of (111) layers stacked alternately; one consists of platinum atoms and the other of copper and platinum atoms arranged regularly along the [110] direction. The structure is shown in Fig. 3. (c) In addition to this ordered phase, the existence of another ordered phase $\mathrm{CuPt}_{7}$ was suggested not by an X-ray diffraction experiment but by an electrical conductivity measurement.

Tang ${ }^{(4)}$ pointed out the inconsistency in their results on $\mathrm{CuPt}_{3}$. That is, Schneider and Esch reported the ordered structure of $\mathrm{CuPt}_{3}$ having an orthorhombic symmetry, but no evidence showing this symmetry
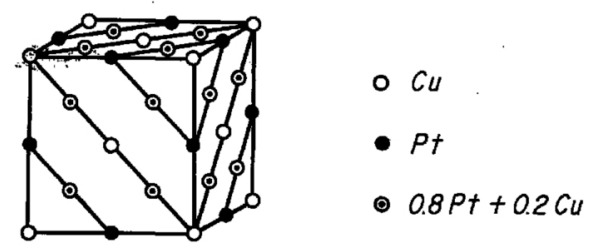

(a)
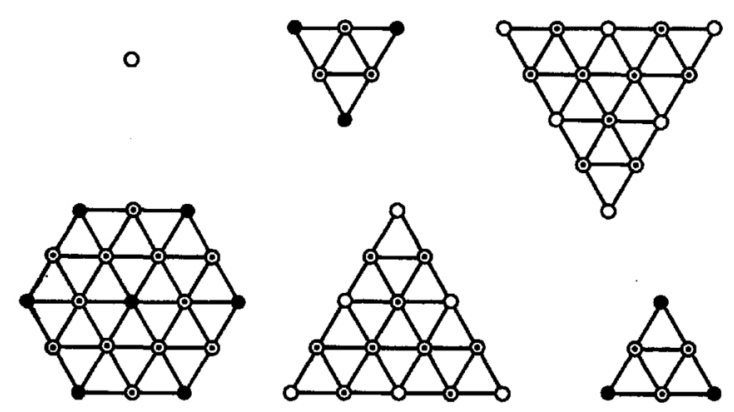

(b)

Fig. 4 Atomic arrangement of the structure model of $\mathrm{CuPt}_{3}$ proposed by Tang.

(4) Y. -C. Tang: Acta Cryst., 4 (1951), 377. was observed in the X-ray diffraction patterns taken by them. Tang proposed a different structure model for $\mathrm{CuPt}_{3}$ which, according to him, gave an intensity distribution in good agreement with the observed one. Figure 4 shows the Tang structure model which has a cubic symmetry.

In the above-mentioned studies, crystal structure analysis was made on the basis of the X-ray powder diffraction patterns, and consequently, owing to the weakness in intensity of superlattice reflections, conflicting results were obtained for the ordered structures of $\mathrm{Cu}_{3} \mathrm{Pt}_{5}$ and $\mathrm{CuPt}_{3}$. In the present work, an X-ray diffraction study has been made using single crystals of the alloys with the compositions ranging from $\mathrm{Cu}-$ $48 \% \mathrm{Pt}$ to $\mathrm{Cu}-70 \% \mathrm{Pt}$ and some well-defined results have been obtained for the ordered structures formed in this composition range.

\section{Experimental Procedures}

Alloys were prepared by melting $99.99 \%$ pure copper and platinum metals in a graphite crucible using a high frequency induction furnace in an argon gas atmosphere. To prevent segregation, the alloy ingots were turned upside down in the crucible and melted again. The lattice constant was measured for the alloy specimens which had been annealed at temperatures higher than $900^{\circ} \mathrm{C}$ and subsequently quenched. By referring to the data on the composition dependence of lattice constant given by Schneider and Esch, the compositions of the alloys prepared were estimated. Chemical analysis was also made for some of the alloy specimens and the results obtained by the lattice constant measurement were confirmed.

Five kinds of the alloy specimens with the following compositions were prepared; $\mathrm{Cu}-48 \% \mathrm{Pt}, \mathrm{Cu}-54 \%$ $\mathrm{Pt}, \mathrm{Cu}-61 \% \mathrm{Pt}, \mathrm{Cu}-66 \% \mathrm{Pt}$ and $\mathrm{Cu}-70 \%$ Pt. Single crystals were grown by melting the alloys in thin ceramic capillaries and solidifying slowly using a moving furnace. Single crystals were readily grown in the alloys containing less platinum, but with increasing platinum content, it became progressively difficult to grow single crystals of good quality. To attain ordered states, the single crystals were annealed at temperatures ranging from 500 to $320^{\circ} \mathrm{C}$. For the alloys with the compositions near the equiatomic one, annealing for several days was shown to be sufficient to develop a fully ordered atomic arrangement, but for the platinum-rich alloys annealing for a period longer than a month was necessary for obtaining appreciable ordering. The single crystals were then chemically thinned in aqua regia to a size $0.25 \mathrm{~mm}$ in diameter in order to reduce an X-ray absorption effect.

Laue photographs were taken to investigate the crystal symmetry and to adjust the crystal orientation. With the $\langle 111\rangle$ direction as a rotation axis, oscillation, rotation and Weissenberg photographs were taken by filtered $\mathrm{CuK} \alpha$ radiation and the intensity distribution in reciprocal space was investigated. Intensities of diffraction spots were estimated by making use of a relation between the X-ray intensity and the blacken- 


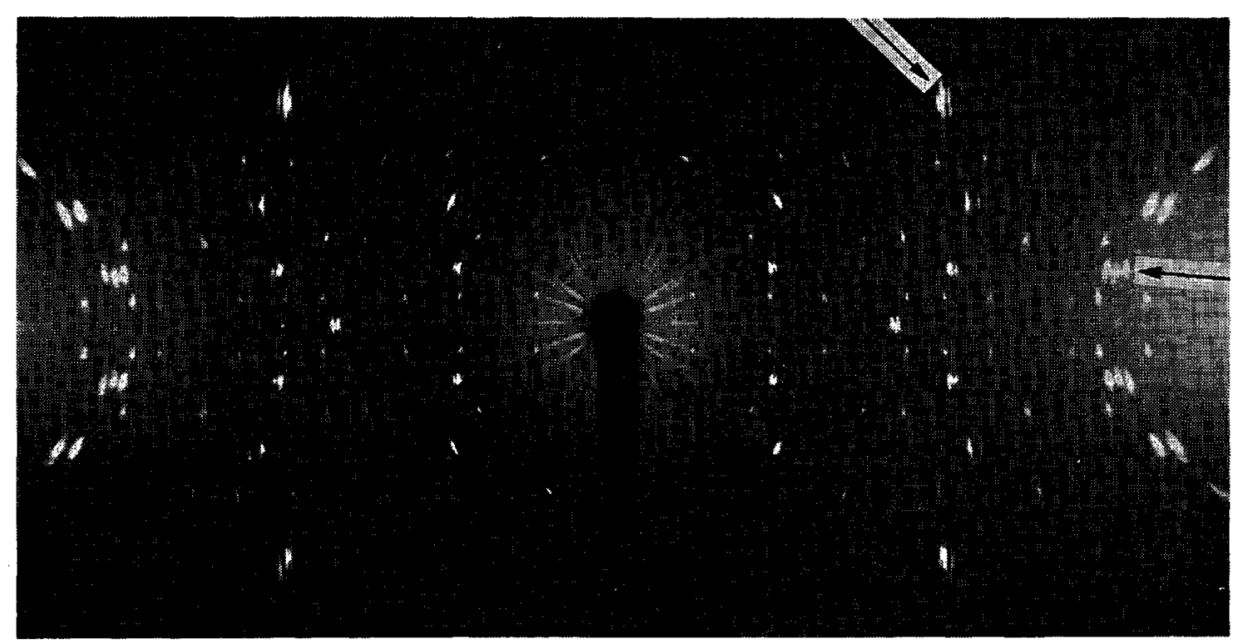

Photo. 1 X-ray rotation photograph taken from the $\mathrm{Cu}-48 \% \mathrm{Pt}$ alloy with $\mathrm{CuK} \alpha$ radiation. 〈111〉 direction was chosen as a rotation axis. Arrows show the split fundamental reflections.

ing of films; the blackening was measured using a microphotometer of the self-balancing type.

\section{Experimental Results and Interpretation of Them}

Photograph 1 shows an X-ray rotation photograph taken from the $\mathrm{Cu}-48 \% \mathrm{Pt}$ alloy annealed at $500^{\circ} \mathrm{C}$ for five days. A similar photograph was obtained for the $\mathrm{Cu}-54 \% \mathrm{Pt}$ alloy. In Photo. 1, intense spots are seen on equatorial, 2nd and 4th layers and weak ones on $1 \mathrm{st}, 3 \mathrm{rd}$ and 5 th layers. The intense spots correspond to fundamental reflections and the weak ones to superlattice reflections.

According to the structure model of $\mathrm{CuPt}$ with a conventional unit cell containing 32 atoms, shown in Fig. 1, there is only one type of superlattice reflection with the structure factor $16\left(f_{\mathrm{Cu}}-f_{\mathrm{Pt}}\right)$ for the equiatomic composition, and the struetere factor of the fundamental reflection is $16\left(f_{\mathrm{Cu}}+f_{\mathrm{Pt}}\right)$. Before comparing the observed intensity distribution with the calculated one, there is one thing to be considered. That is, when ordering takes place in the alloy, it is possible that ordered layers are alternately stacked not only in a particular $\langle 111\rangle$ direction of the cubic lattice but also in the other crystallographically equivalent directions, and there are four possible orientations of the ordered atomic arrangement in the alloy crystal. As mentioned already, the ordered lattice of CuPt is no longer cubic but rhombohedral. The splitting of the fundamental reflections in Photo. 1, shown by the arrows, can be interpreted as being due to the overlapping of diffraction spots from atomic planes with slightly different interplanar spacings. If the existence of the four orientations is taken into account, the intensity distribution deduced from the oscillation and Weissenberg photographs is consistent with that to be expected from the structure model of CuPt. The lattice constants measured for the $\mathrm{Cu}-48 \% \mathrm{Pt}$ alloy are $a=7.554 \AA$ and $\alpha=91.0^{\circ}$.

In Photo. 2 is shown a rotation photograph taken from the $\mathrm{Cu}-61 \% \mathrm{Pt}$ alloy annealed at $350^{\circ} \mathrm{C}$ for two months. This X-ray diffraction pattern resembles that shown in Photo. 1, except that the splitting of the fundamental reflections is not very remarkable. According to the structure model of $\mathrm{Cu}_{3} \mathrm{Pt}_{5}$ proposed by Linde, there should be additional superlattice reflections on the even-order layers in the rotation photograph, besides those corresponding to the CuPt-type

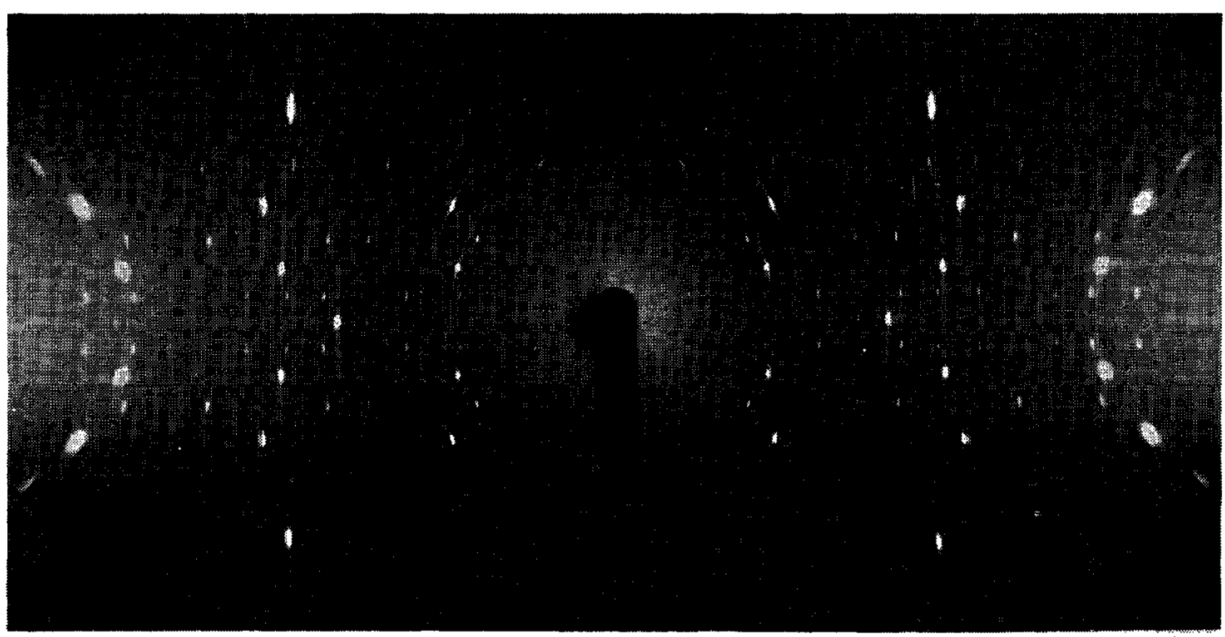

Photo. 2 X-ray rotation photograph taken from the $\mathrm{Cu}-61 \% \mathrm{Pt}$ alloy. 


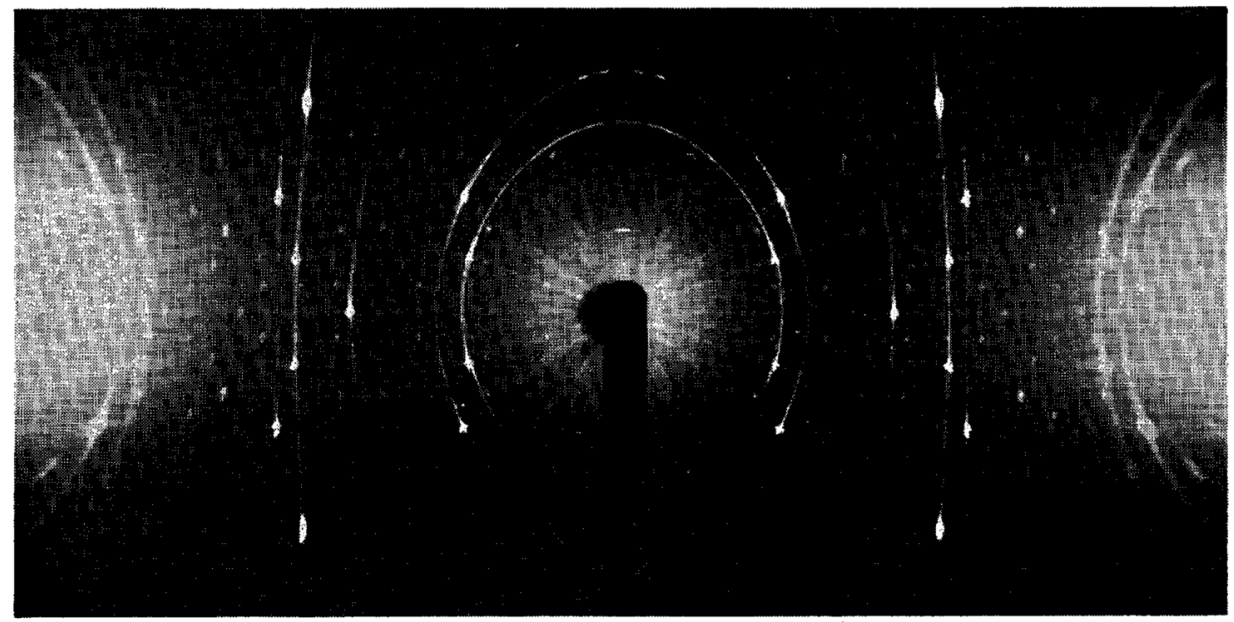

Photo. 3 X-ray rotation photograph taken from the $\mathrm{Cu}-70 \% \mathrm{Pt}$ alloy.

structure. Such additional reflections were not at all observed in the present experiment. The observed intensity distribution of the $\mathrm{Cu}-61 \% \mathrm{Pt}$ alloy is in agreement with that to be expected from the CuPt-type structure with a decreased amount of the lattice distortion. Similar results were obtained for the $\mathrm{Cu}-$ $66 \% \mathrm{Pt}$ alloy. The ordered atomic arrangement in the compositions near $\mathrm{Cu}_{3} \mathrm{Pt}_{5}$ is therefore nothing but of the CuPt type, in which excess platinum atoms replace copper atoms at random in the copper-rich (111) layers. The measured lattice constants for the $\mathrm{Cu}-$ $61 \%$ Pt alloy are $a=7.637 \AA$ and $\alpha=90.37^{\circ}$.

Photograph 3 shows a rotation photograph taken from the $\mathrm{Cu}-70 \% \mathrm{Pt}$ alloy which was annealed at $500^{\circ} \mathrm{C}$ for a month. A significant feature is observed in the photograph, that is, in addition to the reflections to be expected from the CuPt-type structure, a number of weak reflections appear. The intensity distribution deduced from the oscillation and Weissenberg photographs is schematically shown in. Fig. 5, where solid circles and shaded ones represent respectively the fundamental and superlattice reflections to be expected from the CuPt-type structure. Open circles represent the weak reflections. Indices are given in terms of the unit cell containing 32 atoms. The crystal symmetry was observed to be cubic. The lattice constant measured was $7.682 \AA$. As mentioned already there are two kinds of structure model for $\mathrm{CuPt}_{3}$. The Tang model gives an intensity distribution that is in agreement with the present observation. On the other hand, the Schneider-Esch model having an orthorhombic symmetry does not seem to be appropriate. However, if the lattice distortion due to the formation of the orthorhombic ordered phase is assumed to be negligibly small and various orientations of the ordered atomic arrangement with respect to the original lattice are assumed to be equally possible, an intensity distribution quite similar to that of the Tang model is obtained from the Schneider-Esch model. Therefore, this may equally be a possible structure model for $\mathrm{CuPt}_{3}$.

There is a simple way to determine which model is better. According to the Tang model, there are two types of superlattice reffection with the structure factors $4\left(f_{\mathrm{Cu}}-f_{\mathrm{Pt}}\right)$ and 2:4 $\left(f_{\mathrm{Cu}}-f_{\mathrm{Pt}}\right)$. The former will be observed at the positions indicated by the shaded circles in Fig. 5 and the latter at the positions marked by the open circles. Namely, the former will be
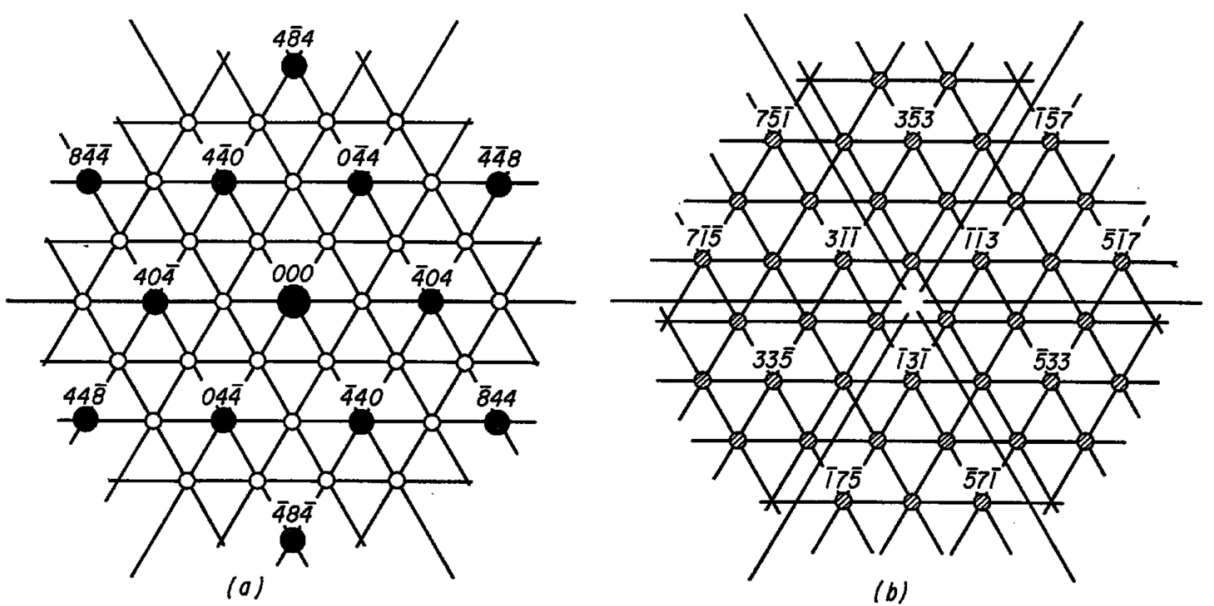

Fig. 5 Observed intensity distribution in reciprocal space of the $\mathrm{Cu}-70 \% \mathrm{Pt}$ alloy. (a) is that on an equatorial layer and (b) a 1st layer. Solid and shaded circles represent respectively the fundamental and superlattice reflections to be expected from the CuPt-type structure, and open circles represent the weak reflections. 
observed on the odd-order layers and the latter on the even order layers in the rotation photograph. For this calculation of the structure factor, platinum content in the alloy was assumed to be $72.5 \%$, because Tang proposed his model at this composition. The ratio of the intensity of the superlattice reflection on the odd-order layer to that on the even order layer should, therefore, be $4^{2} /(2.4)^{2}=2.8$, provided that the other factors affecting the intensity are the same for the two kinds of reflection. This condition may be fulfilled when the reflections with nearly the same Bragg angles are chosen. For the Schneider-Esch model there is only one type of superlattice reflection with the structure factor $7.73\left(f_{\mathrm{Cu}}-f_{\mathrm{Pt}}\right)$, when the platinum content is $72.5 \%$. There are twelve different orientations in total of the ordered atomic arrangement and, if the intensity distribution corresponding to every orientation is superposed, the intensity of the spots at the positions of the shaded circles in Fig. 5 is proportional to $6 \times(7.73)^{2} \times\left(f_{\mathrm{Cu}}-f_{\mathrm{Pt}}\right)^{2}$ and that at the positions of the open circles to $4 \times(7.73)^{2} \times\left(f_{\mathrm{Cu}}-f_{\mathrm{Pt}}\right)^{2}$. Consequently, the intensity ratio would be $6 / 4=1.5$. An advantage of taking the intensity ratio of two superlattice reflections over taking that of a superlattice reflection to a fundamental reflection is that it is not or little dependent upon the degree of atomic order. Measurements of the intensity ratio were made using diffraction spots recorded on oscillation photographs of the $\mathrm{Cu}-70 \% \mathrm{Pt}$ alloy. Although there is a scatter in the measured values owing to unavoidable errors, an average of the values is 2.5 , showing that the Tang model is better. A small difference between the composition of the alloy used in the measurement, $70 \% \mathrm{Pt}$, and that assumed in the calculation of the structure factor, $72.5 \% \mathrm{Pt}$, is not considered to affect significantly the above conclusion.

\section{Discussion}

The structure model for $\mathrm{Cu}_{3} \mathrm{Pt}_{5}$ proposed by Linde seems to be a plausible one, since its atomic arrange- ment is derivable in a simple way from that of CuPt without changing the symmetry of crystal lattice. However, its existence has not been confirmed by the present work. There have been no reports on the existence of $A_{3} B_{5}$-type ordered structure in the facecentered cubic solid solutions without miscibility gap and the copper-platinum alloy system has not been proved to be an exception of the general empirical findings.

For $\mathrm{CuPt}_{3}$ the Tang model has been shown to be superior to the Schneider-Esch one. Then, there remains a problem to determine an exact range in the phase diagram over which the Tang structure is stable, but this remains as a subject of future work. In connection with the ordered structure of $\mathrm{CuPt}_{3}$, it is of interest to know what type of an ordered atomic arrangement is formed on the copper-rich side of the present alloy system. According to a recent work of Ogawa, Iwasaki and Terada ${ }^{(5)}$, at the composition near $\mathrm{Cu}_{3} \mathrm{Pt}$, an $\mathrm{Ll}_{2}$ type structure is formed below $430^{\circ} \mathrm{C}$ and above this temperature it changes into the one-dimensional long period structure which is stable up to $560^{\circ} \mathrm{C}$. The ordered structures formed at the compositions $A_{3} B$ and $A_{3}$ are not isomorphous to each other in the copper-platinum alloy system, but they are isomorphous in other alloy systems as is the case with the copper-gold system. More work is required to clarify the cause for such different behaviors.

A study of the ordered structure of $\mathrm{CuPt}_{7}$ was not made in the present work, for it was not possible to grow single crystals of good quality from the alloy with this composition. Unlike the case of X-ray diffraction, crystal grains of submicron size are sufficient to produce single crystalline patterns for electron diffraction, if the selected area diffraction method is used. Therefore, this method of investigation may be suitable for studying such an ordered structure.

(5) S. Ogawa, H. Iwasaki and A. Terada: J. Phys. Soc. Japan, 34 (1973), 384. 